\title{
Hipertricose como Marcador Subfenotípico de Retardo Neurológico e Neuromotor
}

\author{
Hypertrichosis as a Subphenotypic Marker of Neurological And Neuromotor Retardation \\ Hipertricosis como Marcador Subfennotípico de Retraso Neurológico Y Neuromotor
}

Recebido: 29/03/2021 | Revisado: 04/04/2021 | Aceito: 08/04/2021 | Publicado: 19/04/2021

\author{
Mirelle Estéfane de Oliveira Caixeta \\ ORCID: https://orcid.org/0000-0001-8183-7301 \\ Centro Universitário de Patos de Minas, Brasil \\ mirellecaixeta@gmail.com \\ Caroline Rodrigues Dias \\ ORCID: https://orcid.org/0000-0002-4777-5829 \\ Centro Universitário de Patos de Minas, Brasil \\ carolinerodrigues@unipam.edu.br \\ Thiago de Amorim Carvalho \\ ORCID: https://orcid.org/0000-0003-1153-0931 \\ Centro Universitário de Patos de Minas, Brasil \\ thiagocarvalho@unipam.edu.br \\ Ivania Aparecida Pimenta Santos Silva \\ ORCID: https://orcid.org/0000-0001-7012-8497 \\ Centro Universitário de Patos de Minas, Brasil \\ ivaniapimenta@unipam.edu.br \\ Rodrigo Soares de Andrade \\ ORCID: https://orcid.org/0000-0001-6114-0929 \\ Centro Universitário de Patos de Minas, Brasil \\ rodrigosa@unipam.edu.br
}

\begin{abstract}
Resumo
O objetivo do estudo foi investigar na literatura disponível, trabalhos que apresentam a correlação de hipertricose congênita com alterações craniofaciais e envolvimento neurológico e neuromotor em pacientes pediátricos e adultos. A pesquisa foi realizada nas bases de dados Online Mendelian Inheritance in Man (OMIM), Protein e com busca ampliada no Pubmed-NCBI, não foi estabelecido período limítrofe quanto a data de publicação. Os descritores empregados na língua inglesa foram "Hypertrichosis", "Neurodevelopmental Disorders" e "Craniofacial Abnormalities". Foram incluídos apenas trabalhos sobre síndromes que tinham associação entre hipertricose, anomalias craniofaciais, neurológicas e neuromotoras. As que não se encaixavam nesses critérios foram excluídas, como trabalhos abordando somente malformações, alterações congênitas e quando citava o Hirsutismo isolado. A pesquisa no OMIM resultou 148 condições que mencionavam hipertricose, destas apenas 27 se encaixaram nos critérios de inclusão. Nota-se características de alterações gerais e de cabeça e pescoço que foram comumente encontradas, entre elas: Hipertricose, deficiência intelectual, retardo mental, palato altamente arqueado, hiperplasia gengival e orelhas dismórficas. Portanto, é de suma importância que o odontólogo esteja atento, saiba identificar e documentar alterações somáticas e faciais, a fim de auxiliar na identificação e confirmação de síndromes, como também proporcionar um diagnóstico mais preciso e melhor desfecho para tratamento das alterações da cavidade bucal.
\end{abstract}

Palavras-chave: Anomalias craniofaciais; Hipertricose; Distúrbios Neurológicos.

\begin{abstract}
The aim of the study was to investigate the available literature, papers that present the correlation of congenital hypertrichosis with craniofacial changes and neurological and neuromotor involvement in pediatric and adult patients. The search was carried out in the Online Mendelian Inheritance in Man (OMIM), Protein and Pubmed-NCBI databases, and no limiting period was established as to the date of publication. The descriptors used in English were "Hypertrichosis", "Neurodevelopmental Disorders" and "Craniofacial Abnormalities". Only papers on syndromes that had an association between hypertrichosis and craniofacial, neurological, and neuromotor abnormalities were included. Those that did not fit these criteria were excluded, such as papers addressing only malformations, congenital abnormalities, and when isolated Hirsutism was mentioned. The OMIM search resulted in 148 conditions that mentioned hypertrichosis, of these only 27 met the inclusion criteria. It is noted that characteristics of general and head and neck alterations were commonly found, among them: Hypertrichosis, intellectual disability, mental retardation, highly arched palate, gingival hyperplasia, and dysmorphic ears. Therefore, it is of utmost importance that the dentist be attentive, know how to identify and document somatic and facial alterations, in order to help in the identification and confirmation of syndromes, as well as to provide a more accurate diagnosis and better outcome for the treatment of the alterations in the oral cavity.
\end{abstract}


Keywords: Hypertrichosis; Neurodevelopmental Disorders; Craniofacial Abnormalities.

\section{Resumen}

El objetivo del estudio fue investigar en la literatura disponible, los trabajos que presentan la correlación de la hipertricosis congénita con las alteraciones craneofaciales y el compromiso neurológico y neuromotor en pacientes pediátricos y adultos. La búsqueda se realizó en las bases de datos Online Mendelian Inheritance in Man (OMIM), Protein y con una búsqueda ampliada en Pubmed-NCBI. Los descriptores utilizados en inglés fueron "Hypertrichosis", "Neurodevelopmental Disorders" y "Craniofacial Abnormalities". Sólo se incluyeron los trabajos sobre síndromes que presentaban una asociación entre hipertricosis y anomalías craneofaciales, neurológicas y neuromotoras. Se excluyeron los que no se ajustaban a estos criterios, como los trabajos que sólo abordaban malformaciones, anomalías congénitas y cuando se mencionaba el hirsutismo aislado. La búsqueda en OMIM dio como resultado 148 enfermedades que mencionaban la hipertricosis, de las cuales sólo 27 cumplían los criterios de inclusión. Observamos características de las alteraciones generales y de la cabeza y el cuello que se encontraron comúnmente, entre ellas: Hipertricosis, discapacidad intelectual, retraso mental, paladar muy arqueado, hiperplasia gingival y orejas dismórficas. Por lo tanto, es de suma importancia que el odontólogo esté atento, sepa identificar y documentar los cambios somáticos y faciales para ayudar en la identificación y confirmación de los síndromes, así como proporcionar un diagnóstico más preciso y un mejor resultado para el tratamiento de los cambios en la cavidad oral.

Palabras clave: Anomalías craneofaciales; Hipertricosis; Trastornos neurológicos.

\section{Introdução}

O cabelo é uma estrutura relevante para o corpo na qual desempenha diversas funções fisiológicas, porém, anormalidades na distribuição e no seu crescimento ocasionalmente acontecem e podem levar a diversos problemas psicossociais (Choi, 2018). O crescimento demasiado de pelo corporal em determinadas áreas do corpo sem dependência androgênica é definido como hipertricose (Pavone, et al., 2015; Ploydaeng, et al., 2019).

O hirsutismo refere-se ao crescimento exagerado de pelos especialmente em mulheres e em locais com dependência androgênica (Pavone, et al., 2015; Saleh, et al., 2020). Comumente, essa condição pode ser confundida com hipertricose verdadeira, logo deve-se fazer a distinção dessas doenças por se tratar de duas condições diferentes (Pavone, et al., 2015).

O comparecimento do folículo piloso na área é base essencial para a existência do pelo, salvando locais como lábios, palma das mãos e sola dos pés no qual se ausentam de pelagem mesmo em quadro hipertricótico (Pavone, et al., 2015; Hayashi, et al., 2017). A hipertricose é classificada em diversas formas, tanto pela sua base etiológica (adquirida ou congênita) quanto pela sua localização (localizada ou generalizada) (Trüeb, 2008). Ademais, a classificação clínica pode ser feita de acordo com o pelo que prevalece- Lanugo, velo ou pelo terminal (Trüeb, 2008; Saleh, et al., 2020; Hafsi \& Badri, 2020; Hoover, et al., 2020).

O conhecimento do mecanismo da hipertricose congênita ainda é limitado pela literatura (Wendelin, et al., 2003). Acredita-se que, ocasionalmente, alterações podem ocorrer no ciclo de crescimento fisiológico do cabelo, especificamente na fase ativa do pelo (anágena), onde os folículos permanecem por tempo maior que o normal, resultando então no fenótipo de hipertricose (Wendelin, et al., 2003; Burg, et al., 2003). Na forma idiopática da condição congênita, o atavismo seria a explicação plausível (Pavone, et al., 2015).

Muitas das vezes o quadro hipertricótico pode não apresentar somente como uma característica primária, mas secundária a uma alteração sistêmica subjacente uma vez que tem elevada incidência ao associar-se a síndromes (Pavone, et al., 2015; Wendelin, et al., 2003; Gilhooley, et al., 2017). Além de distúrbios sistêmicos e do neurodesenvolvimento, foram comumente encontradas na literatura disponível alterações de cabeça e pescoço nesses portadores de hipertricose congênita associadas a síndromes específicas, incluindo anormalidades oculares, microcefalia e pescoço curto, e alterações dentárias como retardos na erupção dentária, atrofia palatal e mudanças na conformação fisiológica da gengiva (Pavone, et al., 2015; Maza, et al., 2009). 
O objetivo do presente trabalho é fornecer uma breve literatura a respeito das síndromes associadas à Hipertricose congênita com problemas de neurodesenvolvimento e com alterações da região de cabeça e pescoço em pacientes pediátricos e adultos.

\section{Metodologia}

O presente estudo consiste em um trabalho de pesquisa exploratório de natureza qualitativa. Baseado na literatura científica, foi feita uma extensa busca a fim de investigar e incluir todos os trabalhos que apresentavam síndromes acompanhadas de Hipertricose congênita com comorbidades craniofaciais e envolvimento neurológico e neuromotor, não estabelecendo limites quanto a data que os mesmos foram publicados.

Foram excluídos aqueles que se tratavam de hirsutismo isolado, hipertricose adquirida, quando não apresentava o envolvimento craniofacial ou quando não acometia o sistema neurológico e neuromotor, além dos polimorfismos. Foi utilizado os descritores ingleses "hypertrichosis" "Neurodevelopmental Disorders" e "Craniofacial Abnormalities" uma vez que a literatura incluída foi somente a inglesa. As bases de dados utilizada foi Online Mendelian Inheritance in Man (OMIM), Protein e com busca ampliada no Pubmed- NCBI.

Foram utilizados artigos base na qual a metodologia mostra que a utilização das bases de dados OMIM e Protein, podem ser consideradas de grande valia na busca por patologias que apresentam um componente genético e que podem estar associadas a alterações de estruturas que são de suma importância para o cirurgião dentista, na qual devem ser pautadas, sintetizadas e reconhecidas pelo mesmo (Brooks, 2018; Carvalho, et al., 2020).

\section{Resultados e Discussão}

A pesquisa no OMIM resultou em 148 estudos na qual mencionavam a hipertricose. Apenas 27 desses estudos foram incluídos pois apresentavam síndromes associadas à hipertricose congênita com algum distúrbio neurológico e neuromotor e com o envolvimento de cabeça e pescoço. As características de alterações gerais e de cabeça e pescoço comumente encontradas nesses pacientes foram: Orelhas dismórficas (15/27), retardo mental (14/27), deficiência intelectual (11/27), palato altamente arqueado (9/27) e modificações na morfologia gengival, incluindo hiperplasia, hipertrofia e fibromatose (8/27).

Problemas neurológicos são habitualmente encontrados concomitantemente a aparição da hipertricose congênita em síndromes específicas. A explicação é que, embriologicamente, o ectoderma origina o sistema nervoso e a pele, assim a deficiência intelectual pode estar fortemente associada a presença dessas manifestações cutâneas congênitas (Pezzani, et al., 2015).

Das 27 síndromes específicas englobando problemas do neurodesenvolvimento, pode-se citar a Síndrome de Wiedemann-Steiner mencionando deficiência intelectual como características frequentemente relatada, Síndrome de Cantu mostrando atraso de desenvolvimento motor em consequência da hipotonia muscular presente nesses doentes além de associação com retardo mental leve, e Síndrome de Zimmermann- Laband com sua heterogeneidade genética caracterizadas por deficiência intelectual com ou sem epilepsia (Beighton, 1970; Cantú, et al., 1982; Abo-Dalo, et al., 2008; Bauer, 2019).

A hipertricose na sua forma generalizada foi comumente encontrada nos estudos avaliados, aparecendo em 21 das síndromes, ao contrário da sua forma localizada que apareceu em apenas 6, porém isso não exclui o fato de que ambas estão associadas ao acometimento do complexo craniofacial, como mostra nas síndromes de Warburg Micro 1 e Warburg Micro 3, Okamoto, Coffin-Siris 8, Zimmermann-Laband 2 e Trichohepatoneurodevelopmental.

Nota-se que o envolvimento da população pediátrica com distúrbios do cabelo é citado na literatura. Nesses casos, há uma dificuldade maior na identificação de distúrbios sistêmicos subjacentes baseados somente nos achados clínicos de hipertricose, visto que uma grande variação da pelagem corporal infantil é aceita como normal até a primeira infância (Wade \& 
Sinclair, 2002). O envolvimento pediátrico foi considerado maior quando relacionado aos pacientes adultos, das 27,16 síndromes apresentaram envolvimento dessa classe isoladamente.

Em relação aos resultados envolvendo a população pediátrica, mais estudos se tornam necessários para compreender melhor a distinção quando uma criança apresenta o quadro hipertricótico ou somente quando apresenta uma pelagem anormalmente grande, porém incluída dentro dos aspectos normais para a faixa etária em questão. Dessa forma, contribuirá com o auxílio do diagnóstico quando há um envolvimento sistêmico genético e com o aprendizado total dos profissionais da área da saúde.

A fim de aumentar o conhecimento clínico dessas síndromes e suas alterações, são fornecidos um resumo descritivo baseados na presença de hipertricose congênita com envolvimento craniofacial e alterações do neurodesenvolvimento, anexados no Quadro 1.

Quadro 1. Síndromes genéticas reportadas com hipertricose e com retardo neurológico e neuromotor: Características.

\begin{tabular}{|c|c|}
\hline Síndromes & Características \\
\hline Cahmr & $\begin{array}{l}\text { Geral: Catarata lamelar congênita, hipertricose generalizada, pectus excavatum e retardo mental. } \\
\text { C\&P: Palato altamente arqueado, microdontia e ponte nasal deprimida. }\end{array}$ \\
\hline Barber-Say & $\begin{array}{l}\text { Geral: Pele seca, hipertricose generalizada e retardo mental. } \\
\text { C\&P: Prognatismo mandibular, micrognatia, orelhas dismórficas, ectrópio palpebral, telecanto, hipertelorismo, nariz } \\
\text { bulboso, nasa hipoplásica, macrostomia, palato arqueado alto e erupção retardada. }\end{array}$ \\
\hline $\begin{array}{l}\text { Fontaine } \\
\text { Progeroid }\end{array}$ & $\begin{array}{l}\text { Geral: Anormalidades no cabelo, aparência envelhecida, falanges distais hipoplásicas, hipertricose, atraso } \\
\text { psicomotor, atraso no desenvolvimento motor, devido à fraqueza muscular, hidrocefalia, hipotonia, hipoplasia do } \\
\text { cerebelo. } \\
\text { C\&P: Braquicefalia, turricefalia, microcefalia, face triangular, filtro plano, aparência progeroide, micrognatia, } \\
\text { retrognatia, prognatia, orelhas dismórficas, fissuras palpebrais curtas, hipertelorismo, displasia nasal, microstomia, } \\
\text { palato arqueado alto e oligodontia. }\end{array}$ \\
\hline Coffin Siris 1 & $\begin{array}{l}\text { Geral: Retardo mental, deficiência intelectual, hipertricose e crescimento deficiente. } \\
\text { C\&P: Fácies grosseiras, hipertricose facial, filtro curto, orelhas dismórficas, deficiência visual e auditiva, } \\
\text { estrabismo, ć́lios longos, ponta nasal larga, macrostomia e dentição atrasada. }\end{array}$ \\
\hline $\begin{array}{l}\text { Zimmermann-Laband } \\
3\end{array}$ & 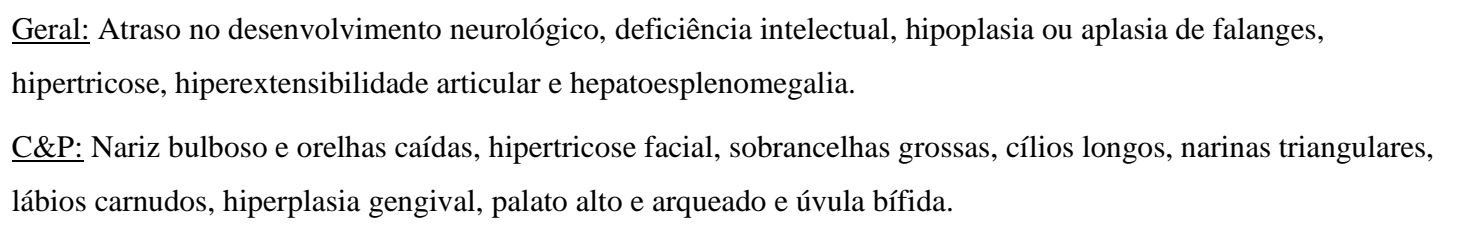 \\
\hline Wiedemann-Steiner & $\begin{array}{l}\text { Geral: Hipertricose cubital, deficiência intelectual e baixa estatura. } \\
\underline{\text { C\&P}} \text { : Face plana, filto longo, orelhas dismórficas, hipertelorismo, estrabismo, sobrancelhas grossas, cílios longos, } \\
\text { ponte nasal ampla, ponta nasal deprimida, palato alto e arqueado, arco de cupido exagerado e dentição anormal. }\end{array}$ \\
\hline Cantu & $\begin{array}{l}\text { Geral: Hipertricose congênita, osteocondrodisplasia, cardiomegalia, linfedema e retardo mental leve. } \\
\text { C\&P: Macrocefalia, fácies grosseiras, testa proeminente, filtro longo, cílios longos, ponte nasal larga e plana, lábios } \\
\text { grossos, hipertrofia gengival e pescoço curto. }\end{array}$ \\
\hline
\end{tabular}




\begin{tabular}{|c|c|}
\hline $\begin{array}{c}\text { Zimmermann-Laband } \\
1\end{array}$ & $\begin{array}{l}\text { Geral: Hipoplasia ou aplasia de falanges, hipertricose, hiperextensibilidade articular, hepatoesplenomegalia e } \\
\text { deficiência intelectual. } \\
\text { C\&P: Nariz bulboso, fácies grosseiras, prognatismo, orelhas dismórficas, perda auditiva, sobrancelhas grossas, } \\
\text { miopia, cataratas, ponte nasal larga, lábios grossos, fibromatose gengival, hiperplasia gengival, palato alto e } \\
\text { arqueado e erupção dentária retardada. }\end{array}$ \\
\hline $\begin{array}{c}\text { Schinzel-Giedion } \\
\text { Midface Retraction }\end{array}$ & $\begin{array}{l}\text { Geral: Hipertricose, retardo mental, anormalidades esqueléticas, malformações geniturinárias, renais e cardíacos. } \\
\text { C\&P: Sutura metópica se estende até a raiz nasal, fácies grosseiras, testa alta e saliente, hipoplasia da face média, } \\
\text { hemangioma facial, orelhas baixas, órbitas rasas, proptose, hipertelorismo, nariz curto, ponte nasal baixa, } \\
\text { macroglossia e pescoço curto. }\end{array}$ \\
\hline Leigh & $\begin{array}{l}\text { Geral: Hipertricose, retardo psicomotor, retardo mental, hipotonia, ataxia, convulsões e disfagia. } \\
\underline{\text { C\&P: Oftalmoplegia, atrofia óptica, nistagmo, estrabismo, ptose e retinopatia pigmentar. }}\end{array}$ \\
\hline Seckel 9 & $\begin{array}{l}\text { Geral: Retardo de crescimento, microcefalia (aspecto cabeça de pássaro), retardo mental e hipertricose. } \\
\underline{\text { C\&P: }} \text { Microcefalia, escafocefalia, rosto comprido e estreito, micrognatia, ouvidos proeminentes e nariz bicudo. }\end{array}$ \\
\hline $\begin{array}{c}\text { Zimmermann-Laband } \\
2\end{array}$ & 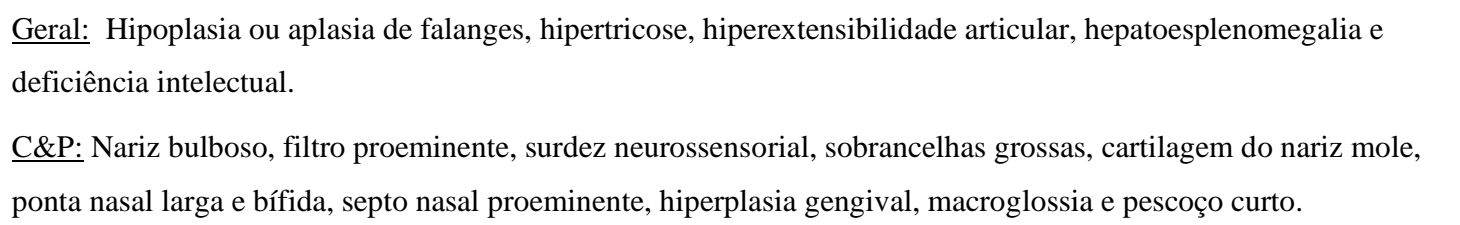 \\
\hline Coffin-Siris 3 & 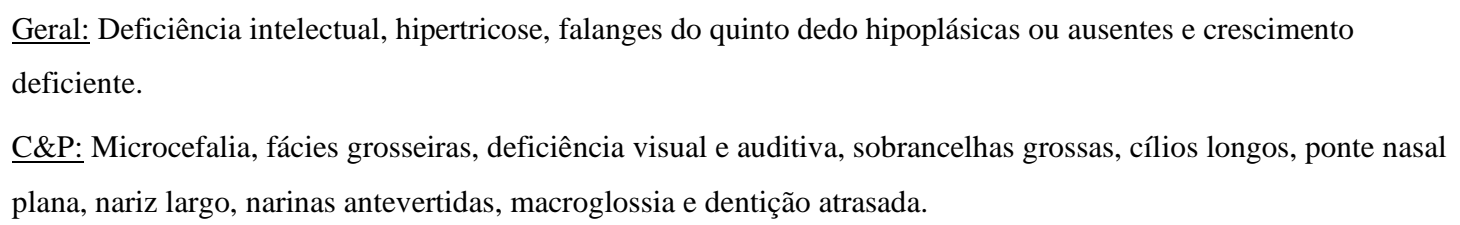 \\
\hline Warburg Micro 1 & $\begin{array}{l}\text { Geral: Baixa estatura, displasia cortical, retardo mental grave, diplegia espástica e hipogonadismo. } \\
\text { C\&P: Microcefalia, micrognatia, orelhas grandes, microftalmia, microcornea, catarata congênita, atrofia óptica, } \\
\text { ptose, olhos profundos e hipertricose facial. }\end{array}$ \\
\hline $\begin{array}{c}\text { Trichohepatoneurode } \\
\text { velopmental }\end{array}$ & $\begin{array}{l}\text { Geral: Baixo peso, cabelo lanoso, disfunção hepática, hipotonia, atraso de desenvolvimento neurológico e } \\
\text { hipertricose. } \\
\text { C\&P: Microcefalia, braquicefalia, plagiocefalia, ptose, hipertelorismo, deficiência visual, hipermetropia, hipoplasia } \\
\text { dos ossos nasais, ponta nasal bulbosa, palato alto e arqueado, macroglossia, diastemas severos, apinhamento } \\
\text { dentário, microdontia e prognatismo. }\end{array}$ \\
\hline Coffin-Siris 4 & $\begin{array}{l}\text { Geral: Deficiência intelectual, hipertricose e crescimento deficiente. } \\
\underline{\text { C\&P}} \text { : Microcefalia, deficiência auditiva e visual, sobrancelhas grossas, cílios longos, ponte nasal plana, nariz largo, } \\
\text { narinas antevertidas, macrostomia, macroglossia e dentição atrasada. }\end{array}$ \\
\hline Warburg Micro 3 & $\begin{array}{l}\text { Geral: Retardo mental grave, diplegia espástica, hipogonadismo e hipertricose. } \\
\text { C\&P: Microcefalia, braquicefalia, filtro curto, micrognatia, orelhas grandes, catarata congênita, microcórnea, } \\
\text { microftalmia, atrofia óptica, nistagmo, blefarofimose, nariz curto, cantos da boca virados para baixo e cristas } \\
\text { alveolares secundárias proeminentes. }\end{array}$ \\
\hline Sandestig-Stefanova & $\begin{array}{l}\text { Geral: Trigonocefalia, camptodactilia, mielinização retardada, deficiência intelectual grave e hipertricose. } \\
\text { C\&P: Microcefalia, trigonocefalia, retrognatia, orelhas baixas, trago hipoplásico, sobrancelhas arqueadas, epicanto, }\end{array}$ \\
\hline
\end{tabular}




\begin{tabular}{|c|c|}
\hline & catarata, microftalmia, ampla ponte e crista nasal, fissura labial e palatina, palato alto e arqueado e pescoço curto. \\
\hline Coffin-Siris 2 & $\begin{array}{l}\text { Geral: Deficiência intelectual, crescimento deficiente e hipertricose. } \\
\underline{\text { C\&P}} \text { : Fácies grosseiras, orelhas malformadas, deficiência visual e auditiva, sobrancelhas grossas, cílios longos, } \\
\text { ponte nasal plana, nariz largo, narinas antevertidas, macrostomia, macroglossia e dentição atrasada. }\end{array}$ \\
\hline Marshall-Smith & $\begin{array}{l}\text { Geral: Maturação esquelética acelerada, dificuldades respiratórias, retardo mental e hipertricose. } \\
\underline{\text { C\&P}} \text { : Testa proeminente, micrognatia, hipoplasia da face média, filtro curto, retrognatia, surdez, orelhas baixas, } \\
\text { olhos proeminentes, esclera azulada, sobrancelhas espessas, nariz curto e empinado, ponte nasal plana, glossoptose, } \\
\text { hipertrofia gengival e dentição irregular. }\end{array}$ \\
\hline Bloom & $\begin{array}{l}\text { Geral: Deficiência de crescimento, pele telangiectásica, retardo mental leve e hipertricose. } \\
\underline{\text { C\&P: Dolicocefalia, microcefalia, hipoplasia malar, nariz proeminente e ausência de incisivos laterais superiores. }}\end{array}$ \\
\hline Rabson-Mendenhall & $\begin{array}{l}\text { Geral: Diabetes mellitus resistente à insulina com hiperplasia pineal, anormalidades somáticas, anomalidades } \\
\text { cutâneas, atraso no desenvolvimento neurológico e hipertricose. } \\
\text { C\&P: Facies grosseira, prognatismo, macroglossia, língua fissurada, hipoplasia gengival, palato altamente arqueado, } \\
\text { displasia dentária, erupção prematura dos dentes e anomalidades dentárias. }\end{array}$ \\
\hline Hunter & $\begin{array}{l}\text { Geral: Deformidades esqueléticas, hepatoesplenomegalia, atraso no desenvolvimento neurológico, hiperatividade, } \\
\text { retardo mental, demência e hipertricose. } \\
\underline{\text { C\&P: Escafocefalia, macrocefalia, papiledema, pigmentação da retina, ptose, macroglossia, erupção dentária }} \\
\text { retardada, dentes amplamente espaçados e pescoço curto. }\end{array}$ \\
\hline Berardinelli-Seip & $\begin{array}{l}\text { Geral: Escassez de tecido adiposo, hipertrigliceridemia, esteatose hepática, retardo mental e hipertricose. } \\
\underline{\text { C\&P: Prognatismo, fácies triangular, aparência acromegalóide e orelhas grandes. }}\end{array}$ \\
\hline Gingival Overgrowth & $\begin{array}{l}\text { Geral: Atraso no desenvolvimento motor e intelectual, convulsões e hipertricose generalizada. } \\
\underline{\text { C\&P}} \text { : Fácies hipotônica, micrognatia, olhos fundos, sobrancelhas grossas e cílios longos, orelhas baixas, filtro } \\
\text { profundo curto, crescimento gengival, vermelhão labial proeminentes e lábio superior evertido. }\end{array}$ \\
\hline Okamoto & $\begin{array}{l}\text { Geral: Hidronefrose congênita, retardo mental grave e deficiência de crescimento. } \\
\text { C\&P: Fenda palatina, hipoplasia do meio da face, hipertricose, cílios longos, olhos proeminentes, epicanto, orelhas } \\
\text { dismórficas, nariz curto arrebitado e pescoço alado. }\end{array}$ \\
\hline Coffin-Siris 8 & $\begin{array}{l}\text { Geral: Deficiência intelectual e falanges do quinto dedo hipoplásicas ou ausentes. } \\
\text { C\&P: Fácies ásperas, hipertricose, sobrancelhas grossas, cílios longos, ptose, narinas antevertidas, anormalidades } \\
\text { oftalmológicas e nariz arrebitado. }\end{array}$ \\
\hline
\end{tabular}

C\&P (Cabeça e pescoço). Fonte: Autores.

É de grande valia atentar-se que todas as síndromes possuem um componente neurológico e neuromotor associado, além da presença da hipertricose, seja ela localizada ou generalizada. Torna-se relevante a análise criteriosa do quadro na divisão "Cabeça \& Pescoço", na qual mostra-se na forma descritiva as principais alterações do complexo craniofacial e buco dental, que são de extrema importância o conhecimento pelos profissionais de saúde oral.

\section{Conclusão}

A identificação e a documentação das anormalidades somáticas e fácies pelo odontólogo é de suma importância, posto que ajudará na identificação e confirmação de síndromes que eventualmente aparecem subjacente aos achados dismórficos craniofaciais (Brooks, 2018). Com isso, proporcionará um diagnóstico mais preciso e oportuno em relação as alterações da cavidade bucal, melhor desfecho clínico para as anormalidades gengivais, periodontais e dentais; e adoção de uma abordagem 
clínica mais adequada para aqueles com problemas neurológicos uma vez que apresentam significativos reflexos orofaciais (Brooks, 2018; Carvalho, et al., 2020; Baptista, 2017).

No geral, apesar das síndromes associadas a hipertricose com envolvimento neurológico e neuromotor serem consideradas de acometimento raro e, juntamente a literatura estritamente limitada acerca desse assunto, estima-se que seja necessário a confecção de mais trabalhos futuros no intuito de contribuir com maiores informações acerca desse assunto, visto que uma grande parte apresenta intimamente associada ao acometimento do complexo craniofacial.

\section{Referências}

Abo-Dalo, B., Roes, M., Canún, S., Delatycki, M., Gillessen-Kaesbach, G., Hrytsiuk, I., Jung, C., Kerr, B., Mowat, D., Seemanova, E., Steiner, C. E., Stewart, H., Thierry, P., Van Buggenhout, G., White, S., Zenker, M., \& Kutsche, K. (2008). No mutation in genes of the WNT signaling pathway in patients with Zimmermann-Laband syndrome. Clinical Dysmorphology, 17(3), p. 181-185. https://doi.org/10.1097/MCD.0b013e3282f2514c

Baptista, H., \& Lopes Cardoso, I. (2017). Steinert syndrome and repercussions in dental medicine. Archives of Oral Biology, 75, p. 37-47. https://doi.org/10.1016/j.archoralbio.2016.12.008

Bauer, C. K., Schneeberger, P. E., Kortüm, F., Altmüller, J., Santos-Simarro, F., Baker, L., Keller-Ramey, J., White, S. M., Campeau, P. M., Gripp, K. W., \& Kutsche, K. (2019). Gain-of-Function Mutations in KCNN3 Encoding the Small-Conductance Ca2+-Activated K+ Channel SK3 Cause Zimmermann-Laband Syndrome. American Journal of Human Genetics, 104(6), p. 1139-1157. https://doi.org/10.1016/j.ajhg.2019.04.012

Beighton, P. H. (1970). Familial hypertrichosis cubiti: hairy elbows syndrome. Journal of Medical Genetics, 7 , p. 158-160. http://dx.doi.org/10.1136/jmg.7.2.158

Brooks, J. K. (2018). A review of syndromes associated with blue sclera, with inclusion of malformations of the head and neck. Oral Surgery, Oral Medicine, Oral Pathology and Oral Radiology, 126(3), p. 252-263. https://doi.org/10.1016/j.oooo.2018.05.012

Burg, D., Yamamoto, M., Namekata, M., Haklani, J., Koike, K., \& Halasz, M. (2017). Promotion of anagen, increased hair density and reduction of hair fall in a clinical setting following identification of FGF5-inhibiting compounds via a novel 2-stage process. Clinical, Cosmetic and Investigational Dermatology, 10, p. 71-85. https://doi.org/10.2147/CCID.S123401

Cantú, J. M., Garcia-Cruz, D., Sánchez-Corona, J., Hernández, A., \& Nazará, Z. (1982). A distinct osteochondrodysplasia with hypertrichosisIndividualization of a probable autosomal recessive entity. Journal of Human Genetics, 60(1), p. 36-41. https://doi.org/10.1007/BF00281261

Carvalho, A., Martelli, D., Barbosa, G., Carvalho, M., Swerts, M.S., \& Martelli-Junior, H. (2020). Cafe-au-lait spots as a clinical sign of syndromes. Clinical Pediatrics, p. 1-23.

Choi, B. Y. (2018). Hair-growth potential of ginseng and its major metabolites: A review on its molecular Mechanisms. International Journal of Molecular Sciences, 19(9), p. 2-13. https://doi.org/10.3390/ijms19092703

Gilhooley, E., Gormally, S., Irvine, A., Lynch, S. A., \& Collins, S. (2017). FOXN1 Duplication and Congenital Hypertrichosis. Pediatric Dermatology, 34(2), p. e77-e79. https://doi.org/10.1111/pde.13078

Hafsi, W.; \& Badri, T. (2020). Hirsutism. [s.1.] StatPearls. Retrieved from https://www.ncbi.nlm.nih.gov/books/NBK470417/

Hayashi, R., Yoshida, K., Abe, R., Niizeki, H., \& Shimomura, Y. (2017). First Japanese case of congenital generalized hypertrichosis with a copy number variation on chromosome 17q24. Journal of Dermatological Science, 85(1), p. 63-65. https://doi.org/10.1016/j.jdermsci.2016.10.010

Hoover, E., \& Krishnamurthy, K. (2018). Physiology, Hair. [s.1.] StatPearls. Retrieved from https://www.ncbi.nlm.nih.gov/books/NBK499948/

Maza, A., Gaudy-Marqueste, C., Collet-Vilette, A. M., Joubert, F., Richard, M. A., \& Grob, J. J. (2009). Hypertrichose universelle congénitale. Annales de Dermatologie et de Venereologie, 136(3), p. 300-302. https://doi.org/10.1016/j.annder.2008.06.008

Pavone, P., Praticò, A. D., Falsaperla, R., Ruggieri, M., Zollino M., Corsello, G., \& Neri, G. (2015). Congenital generalized hypertrichosis: The skin as a clue to complex malformation syndromes. In Italian Journal of Pediatrics, 41(1), p. 55. https://doi.org/10.1186/s13052-015-0161-3

Pezzani, L., Milani, D., \& Tadini, G. (2015). Intellectual Disability: When the Hypertrichosis Is a Clue. Journal of Pediatric Genetics, 04(03), p. 154-158. https://doi.org/10.1055/s-0035-1564442

Ploydaeng, M., Rojhirunsakool, S., \& Suchonwanit, P. (2019). Localized hypertrichosis with traumatic panniculitis: A case report and literature review. Case Reports in Dermatology, 11(2), p. 180-186. https://doi.org/10.1159/000501360

Saleh, D., Yarrarapu S. N. S., \& Cook, C. (2020). Hypertrichosis. StatPearls. Retrieved from https://www.ncbi.nlm.nih.gov/books/NBK534854/

Trüeb, R. M. (2008). Hypertrichose. Hautarzt, 59(4), p. 325-338. https://doi.org/10.1007/s00105-008-1489-z

Wade, M. S., \& Sinclair, R. D. (2002). Disorders of hair in infants and children other than alopecia. Clinics in Dermatology, 20(1), p. 16-28. https://doi.org/10.1016/S0738-081X(01)00226-7

Wendelin, D. S., Pope, D. N., \& Mallory, S. B. (2003). Hypertrichosis. Journal of the American Academy of Dermatology, 48, p. 161-182. https://doi.org/10.1067/mjd.2003.100 